\title{
CONFORMALLY FLAT 4-TH ROOT $(\alpha, \beta)$-METRICS WITH RELATIVELY ISOTROPIC MEAN LANDSBERG CURVATURE
}

\author{
AKBAR TAYEBI* AND MARZEIYA AMINI
}

\begin{abstract}
In this paper, we study conformally flat 4 -th root $(\alpha, \beta)$ metrics on a manifold $M$ of dimension $n \geq 3$. We prove that every conformally flat 4 -th root $(\alpha, \beta)$-metric with relatively isotropic mean Landsberg curvature must be either Riemannian metrics or locally Minkowski metrics.
\end{abstract}

MSC(2010): 53B40; 53C60.

Keywords: Exponential metric, conformally flat metric, relatively isotropic mean Landsberg curvature.

\section{Introduction}

The study of Conformal Geometry has a long and venerable history. From the beginning, conformal geometry has played an important role in Physical Theories. The conformal transformation of Riemannian metrics have been well studied by many geometers. The Weyl theorem states that the projective and conformal properties of a Finsler space determine the metric properties uniquely. There are many important local and global results in Riemannian conformal geometry, which in turn lead to a better understanding on Riemann manifolds.

As Chern said Finsler geometry is just Riemannian geometry without the quadratic restriction. Thus the conformal properties of a Finsler metric deserve extra attention. Let $F$ and $\tilde{F}$ be two arbitrary Finsler metrics on a manifold $M$ Then we say that $F$ is conformal to $\tilde{F}$ if and only if there exists a scalar function $\sigma=\sigma(x)$ such that $F(x, y)=e^{\sigma(x)} \tilde{F}(x, y)$. The scalar function $\sigma$ is called the conformal factor.

A Finsler metric $F=F(x, y)$ on a manifold $M$ is called a conformally flat metric if there exists a locally Minkowski metric $\tilde{F}=\tilde{F}(y)$ such that $F=e^{\kappa(x)} \tilde{F}$, where $\kappa=\kappa(x)$ is a scalar function on $M$. A new and hot issue

Date: Received: September 20, 2020 , Accepted: October, 26, 2020.

* Corresponding author. 
is to characterization of conformally flat Finsler metrics. In [2], Asanov constructed a Finslerian metric function on the manifold $N=\mathbb{R} \times M$, where $M$ is a Riemannian manifold endowed with two real functions, and showed that the tangent Minkowski spaces of such a Finsler space are conformally flat. This motivated him to propose a Finslerian extension of the electromagnetic field equations whose solutions are explicit images of the solutions to the ordinary Maxwell equations.

In order to find conformally flat Finsler metrics, we consider the class of $m$-th root Finsler metrics. Let $(M, F)$ be an $n$-dimensional Finsler manifold, $T M$ its tangent bundle and $\left(x^{i}, y^{i}\right)$ the coordinates in a local chart on $T M$. Let $F: T M \rightarrow \mathbb{R}$ be a scalar function defined by $F=\sqrt[m]{A}$, where $A$ is given by $A:=a_{i_{1} \ldots i_{m}}(x) y^{i_{1}} y^{i_{2}} \ldots y^{i_{m}}$ such that $a_{i_{1} \ldots i_{m}}$ is symmetric in all its indices. Then $F$ is called an $m$-th root Finsler metric. An $m$-th root Finsler metric can be regarded as a direct generalization of a Riemannian metric in the sense that the 2-th root metric is a Riemannian metric $F=$ $\sqrt{a_{i j}(x) y^{i} y^{j}}$. The fourth root metrics $F=\sqrt[4]{a_{i j k l}(x) y^{i} y^{j} y^{k} y^{l}}$ are called the quartic metrics. The special quartic metric $F=\sqrt[4]{y^{i} y^{j} y^{k} y^{l}}$ is called Berwald-Moór metric which plays an important role in theory of space-time structure, gravitation and general relativity. For more progress, see [6], [7], [8] and [10].

In [9], the first author and Razgordani proved that every conformally flat weakly Einstein 4 -th root $(\alpha, \beta)$-metric on a manifold $M$ of dimension $n \geq 3$ is either a Riemannian metric or a locally Minkowski metric. Also, they showed that every conformally flat 4 -th root $(\alpha, \beta)$-metric of almost vanishing $\Xi$-curvature on a manifold $M$ of dimension $n \geq 3$ reduces to a Riemannian metric or a locally Minkowski metric. In this paper, we study conformally flat 4 -th root $(\alpha, \beta)$-metric with relatively isotropic mean Landsberg curvature. More precisely, we prove the following.

Theorem 1.1. Let $F=F(x, y)$ be a conformally flat 4 -th root $(\alpha, \beta)$-metric on a manifold $M$ of dimension $n \geq 3$. Suppose that $F$ has relatively isotropic mean Landsberg curvature

$$
\mathbf{J}+c(x) F \mathbf{I}=0,
$$

where $c=c(x)$ is a scalar function on $M$. Then $F$ reduces to a Riemannian metric or a locally Minkowski metric.

\section{Preliminaries}

Let $M$ be a $n$-dimensional $C^{\infty}$ manifold and $T M=\bigcup_{x \in M} T_{x} M$ the tangent bundle. Let $(M, F)$ be a Finsler manifold. The following quadratic form $\mathbf{g}_{y}$ on $T_{x} M$ is called fundamental tensor

$$
\mathbf{g}_{y}(u, v)=\left.\frac{1}{2} \frac{\partial^{2}}{\partial s \partial t}\left[F^{2}(y+s u+t v)\right]\right|_{s=t=0}, \quad u, v \in T_{x} M .
$$

Let $x \in M$ and $F_{x}:=\left.F\right|_{T_{x} M}$. To measure the non-Euclidean feature of $F_{x}$, for a non-zero vector $y \in T_{x} M_{0}:=T_{x} M-\{0\}$, define $\mathbf{C}_{y}: T_{x} M \times T_{x} M \times$ 
$T_{x} M \rightarrow \mathbb{R}$ by

$\mathbf{C}_{y}(u, v, w):=\frac{1}{2} \frac{d}{d t}\left[\mathbf{g}_{y+t w}(u, v)\right]_{t=0}=\frac{1}{4} \frac{\partial^{3}}{\partial r \partial s \partial t}\left[F^{2}(y+r u+s v+t w)\right]_{r=s=t=0}$, where $u, v, w \in T_{x} M$. By definition, $\mathbf{C}_{y}$ is a symmetric trilinear form on $T_{x} M$. The family $\mathbf{C}:=\left\{\mathbf{C}_{y}\right\}_{y \in T M_{0}}$ is called the Cartan torsion. Thus $\mathbf{C}=0$ if and only if $F$ is Riemannian.

For $y \in T_{x} M_{0}$, define $\mathbf{I}_{y}: T_{x} M \rightarrow \mathbb{R}$ by

$$
\mathbf{I}_{y}(u)=\sum_{i=1}^{n} g^{i j}(y) \mathbf{C}_{y}\left(u, \partial_{i}, \partial_{j}\right),
$$

where $\left\{\partial_{i}\right\}$ is a basis for $T_{x} M$ at $x \in M$. The family $\mathbf{I}:=\left\{\mathbf{I}_{y}\right\}_{y \in T M_{0}}$ is called the mean Cartan torsion. Thus, $\mathbf{I}_{y}(u):=I_{i}(y) u^{i}$, where $I_{i}:=g^{j k} C_{i j k}$.

On the slit tangent bundle $T M_{0}$, the Landsberg curvature $\mathbf{L}_{i j k}:=L_{i j k} d x^{i} \otimes$ $d x^{j} \otimes d x^{k}$ is defined by $L_{i j k}:=C_{i j k ; m} y^{m}$, where ";" denotes the horizontal covariant derivative with respect to $F$. Further, the Landsberg curvature can be expressed as following

$$
L_{i j k}=-\frac{1}{2} F F_{y^{m}}\left[G^{m}\right]_{y^{i} y^{j} y^{k}}
$$

A Finsler metric is called the Landsberg metric if $L_{i j k}=0$.

The horizontal covariant derivatives of the mean Cartan torsion $\mathbf{I}$ along geodesics give rise to the mean Landsberg curvature $\mathbf{J}_{y}: T_{x} M \rightarrow \mathbb{R}$ which are defined by $\mathbf{J}_{y}(u):=J_{i}(y) u^{i}$, where

$$
J_{i}:=I_{i \mid s} y^{s} \text {. }
$$

Here, "|" denotes the horizontal covariant derivative with respect to the Berwald connection of $F$. The family $\mathbf{J}:=\left\{\mathbf{J}_{y}\right\}_{y \in T M_{0}}$ is called the mean Landsberg curvature. Also, the mean Landsberg curvature can be expressed as following

$$
J_{i}:=g^{j k} L_{i j k}
$$

A Finsler metric $F$ on a manifold $M$ is called of relatively isotropic mean Landsberg curvature if

$$
\mathbf{J}+c F \mathbf{I}=0,
$$

where $c=c(x)$ is a scalar function on $M$.

In this paper, we will focus on studying regular $(\alpha, \beta)$-metrics. Let "|" denote the covariant derivative with respect to the Levi-Civita connection of $\alpha$. Denote

$$
\begin{gathered}
r_{i j}:=\frac{1}{2}\left(b_{i \mid j}+b_{j \mid i}\right), \quad s_{i j}:=\frac{1}{2}\left(b_{i \mid j}-b_{j \mid i}\right) \\
s_{j}^{i}:=a^{i m} s_{m j}, \quad r_{j}^{i}:=a^{i m} r_{m j}, \quad r_{j}:=b^{i} r_{i j}, \quad s_{j}:=b^{i} s_{i j},
\end{gathered}
$$

where $\left(a^{i j}\right):=\left(a_{i j}\right)^{-1}$ and $b^{j}:=a^{j k} b_{k}$. We put

$$
r_{0}:=r_{i} y^{i}, \quad s_{0}:=s_{i} y^{i}, \quad r_{00}:=r_{i j} y^{i} y^{j}, \quad s_{i 0}:=s_{i j} y^{j} .
$$


Let $G^{i}$ and $G_{\alpha}^{i}$ denote the geodesic coefficients of $F$ and $\alpha$ respectively in the same coordinate system. Then we have

$$
G^{i}=G_{\alpha}^{i}+\alpha Q s_{0}^{i}+\left\{r_{00}-2 Q \alpha s_{0}\right\}\left\{\Psi b^{i}+\Theta \alpha^{-1} y^{i}\right\},
$$

where

$$
\begin{aligned}
Q & :=\frac{\phi^{\prime}}{\phi-s \phi^{\prime}}, \\
\Theta & :=\frac{\phi \phi^{\prime}-s\left(\phi \phi^{\prime \prime}+\phi^{\prime} \phi^{\prime}\right)}{2 \phi\left[\phi-s \phi^{\prime}+\left(b^{2}-s^{2}\right) \phi^{\prime \prime}\right]}, \\
\Psi & :=\frac{\phi^{\prime \prime}}{2\left[\phi-s \phi^{\prime}+\left(b^{2}-s^{2}\right) \phi^{\prime \prime}\right]} .
\end{aligned}
$$

For more details, see [4]. Let

$$
\begin{aligned}
& \Delta:=1+s Q+\left(b^{2}-s^{2}\right) Q^{\prime}, \\
& \Phi:=-(n \Delta+1+s Q)\left(Q-s Q^{\prime}\right)-\left(b^{2}-s^{2}\right)(1+s Q) Q^{\prime \prime}, \\
& \Psi_{1}:=\sqrt{b^{2}-s^{2}} \Delta^{\frac{1}{2}}\left[\frac{\sqrt{b^{2}-s^{2}} \Phi}{\Delta^{\frac{3}{2}}}\right]^{\prime}, \\
& h_{j}:=b_{j}-\alpha^{-1} s y_{j}:
\end{aligned}
$$

By (2.1), (2.2), (2.3), the mean Landsberg curvature of the $(\alpha, \beta)$-metric $F=\alpha \phi(s), s=\beta / \alpha$, is given by

$$
\begin{aligned}
& J_{j}=\frac{1}{2 \alpha^{4} \Delta}\left\{\frac{2 \alpha^{3}}{b^{2}-s^{2}}\left[\frac{\Phi}{\Delta}+(n+1)\left(Q-s Q^{\prime}\right)\right]\left(s_{0}+r_{0}\right) h_{j}\right. \\
& +\frac{\alpha^{2}}{b^{2}-s^{2}}\left[\Psi_{1}+s \frac{\Phi}{\Delta}\right]\left(r_{00}-2 \alpha Q s_{0}\right) h_{j} \\
& +\alpha\left[-\alpha^{2} Q^{\prime} s_{0} h_{j}+\alpha Q\left(\alpha^{2} s_{j}-y_{j} s_{0}\right)+\alpha^{2} \Delta s_{j 0}\right. \\
& \left.\left.+\alpha^{2}\left(r_{j 0}-2 \alpha Q s_{j}\right)-\left(r_{00}-2 \alpha Q s_{0}\right) y_{j}\right] \frac{\Phi}{\Delta}\right\} .
\end{aligned}
$$

Here, $y_{j}=a_{i j} y^{i}$. See [3] and [5].

\section{Proof of Theorem 1.1}

in this section, we are going to prove Theorem 1.1. To prove it, we need the following.

Lemma 3.1. ([3]) For an $(\alpha, \beta)$-metric $F=\alpha \phi(s), s=\beta / \alpha$, the mean Cartan torsion is given by

$$
I_{i}=-\frac{1}{2 F} \frac{\Phi}{\Delta}\left(\phi-s \phi^{\prime}\right) h_{i}
$$

In [3], the following was proved. 
Lemma 3.2. ([3]) An ( $\alpha, \beta)$-metric $F$ is a Riemannian metric if and only if $\Phi=0$.

In [3], the following formula obtained

$$
\begin{array}{r}
J_{j}+c(x) F I_{j}=-\frac{1}{2 \alpha^{4} \Delta}\left\{\frac{2 \alpha^{3}}{b^{2}-s^{2}}\left[\frac{\Phi}{\Delta}+(n+1)\left(Q-s Q^{\prime}\right)\right]\left(s_{0}+r_{0}\right) h_{j}\right. \\
+\frac{\alpha^{2}}{b^{2}-s^{2}}\left[\Psi_{1}+s \frac{\Phi}{\Delta}\right]\left(r_{00}-2 \alpha Q s_{0}\right) h_{j} \\
+\alpha\left[-\alpha^{2} Q^{\prime} s_{0} h_{j}+\alpha Q\left(\alpha^{2} s_{j}-y_{j} s_{0}\right)+\alpha^{2} \Delta s_{j 0}+\alpha^{2}\left(r_{j 0}-2 \alpha Q s_{j}\right)\right. \\
\left.\left.-\left(r_{00}-2 \alpha Q s_{0}\right) y_{j}\right] \frac{\Phi}{\Delta}+c(x) \alpha^{4} \Phi\left(\phi-s \phi_{\prime}\right) h_{j}\right\} .
\end{array}
$$

Also, we remark the following key lemma.

Lemma 3.3. ([1]) Let $F=\alpha \phi(s), s=\beta / \alpha$, be an $(\alpha, \beta)$-metric. Then $F$ is locally Minkowskian if and only if $\alpha$ is a flat Riemannian metric and $\beta$ is parallel with respect to $\alpha$.

Also, the following holds.

Lemma 3.4. ([3]) If $\phi=\phi(s)$ satisfies $\Psi_{1}=0$, then $F$ is Riemannian.

Now, assume that $F=\alpha \phi(s), s=\beta / \alpha$, is a conformally flat Finsler metric, that is, $F$ is conformally related to a Minkowski metric $\tilde{F}$. Then there exists a scalar function $\sigma=\sigma(x)$ on the manifold, so that $\tilde{F}=e^{\sigma(x)} F$. It is easy to see that $\tilde{F}=\tilde{\alpha} \phi(\tilde{s}), \tilde{s}=\tilde{\beta} / \tilde{\alpha}$. We have $\tilde{\alpha}=e^{\sigma(x)} \alpha$ and $\tilde{\beta}=e^{\sigma(x)} \beta$ which are equivalent to

$$
\tilde{a_{i j}}=e^{2 \sigma(x)} a_{i j}, \quad \tilde{b_{i}}=e^{\sigma(x)} b_{i} .
$$

Let "\|" denote the covariant derivative with respect to the Levi-Civita connection of $\tilde{\alpha}$. Put $\sigma_{i}:=\partial \sigma / \partial x^{i}$ and $\sigma^{i}:=a^{i j} \sigma_{j}$. The Christoffel symbols $\Gamma_{j k}^{i}$ of $\alpha$ and the Christoffel symbols $\tilde{\Gamma}_{j k}^{i}$ of $\tilde{\alpha}$ are related by

$$
\tilde{\Gamma}_{j k}^{i}=\Gamma_{j k}^{i}+\delta_{j}^{i} \sigma_{k}+\delta_{k}^{i} \sigma_{j}-\sigma^{i} a_{j k} .
$$

Hence, one can obtain

$$
\tilde{b}_{i \| j}=\frac{\partial \tilde{b}_{i}}{\partial x^{j}}-\tilde{b}_{s} \tilde{\Gamma}_{j k}^{i}=e^{\sigma}\left(b_{i \mid j}-b_{j} \sigma_{i}+b_{r} \sigma^{r} a_{i j}\right) .
$$


By Lemma 3.31, for Minkowski metric $\tilde{F}$, we have $\tilde{b}_{i \| j}=0$. Thus

$$
\begin{aligned}
b_{i \mid j} & =b_{j} \sigma_{i}-b_{r} \sigma^{r} a_{i j}, & & \\
r_{i j} & =\frac{1}{2}\left(\sigma_{i} b_{j}+\sigma_{j} b_{i}\right)-b_{r} \sigma^{r} a_{i j}, & s_{i j} & =\frac{1}{2}\left(\sigma_{i} b_{j}+\sigma_{j} b_{i}\right), \\
r_{j} & =-\frac{1}{2}\left(b_{r} \sigma^{r}\right) b_{j}+\frac{1}{2} \sigma_{j} b^{2}, & s_{j} & =\frac{1}{2}\left(b_{r} \sigma^{r}\right) b_{j}-\sigma_{j} b^{2}, \\
r_{i 0} & =\frac{1}{2}\left[\sigma_{i} \beta+\left(\sigma_{r} y^{r}\right) b_{i}\right]-\sigma_{r} b^{r} y_{i}, & s_{i 0} & =\frac{1}{2}\left[\sigma_{i} \beta+\left(\sigma_{r} y^{r}\right) b_{i}\right] .
\end{aligned}
$$

Further, we have

$r_{00}=\left(\sigma_{r} y^{r}\right) \beta-\left(\sigma_{r} y^{r}\right) \alpha^{2}, \quad r_{0}=\frac{1}{2}\left(\sigma_{r} y^{r}\right) b^{2}-\frac{1}{2}\left(\sigma_{r} b^{r}\right) \beta, \quad s_{0}=\frac{1}{2}\left(\sigma_{r} y^{r}\right) \beta-\frac{1}{2}\left(\sigma_{r} y^{r}\right) b^{2}$.

By (3.8), the conformally flat $(\alpha, \beta)$-metrics satisfying $r_{0}+s_{0}=0$ which is equivalent to the length of $\beta$ with respect to $\alpha$ being a constant. We take an orthonormal basis at any point $x$ with respect to $\alpha$ such that $\alpha=$ $\sqrt{\sum_{i=1}^{n}\left(y^{i}\right)^{2}}$ and $\beta=b y^{1}$, where $b:=\left\|\beta_{x}\right\|_{\alpha}$. By using the same coordinate transformation $\psi:\left(s, u^{A}\right) \longrightarrow\left(y^{i}\right)$ in $T_{x} M$, we get

$$
y_{1}=\frac{s}{\sqrt{b^{2}-s^{2}}} \bar{\alpha}, \quad y^{A}=u^{A}, \quad 2 \leq A \leq n,
$$

where $\bar{\alpha}=\sqrt{\sum_{i=2}^{n}\left(u^{A}\right)^{2}}$. We have

$$
\alpha=\frac{b}{\sqrt{b^{2}-s^{2}}} \bar{\alpha}, \quad \beta=\frac{b s}{\sqrt{b^{2}-s^{2}}} \bar{\alpha} .
$$

Put $\overline{\sigma_{0}}:=\sigma_{A} u^{A}$. Then, by (3.5)-(3.7), (3.9) and (3.10) we have

$$
\begin{aligned}
& r_{00}=-b \sigma_{1} \bar{\alpha}^{2}+\frac{b s \overline{\sigma_{0}} \bar{\alpha}}{\sqrt{b^{2}-s^{2}}}, \quad r_{0}=\frac{1}{2} b^{2} \overline{\sigma_{0}}=-s_{0}, \quad r_{10}=\frac{1}{2} b \overline{\sigma_{0}}, \\
& r_{A 0}=\frac{1}{2} \frac{\sigma_{A} b s \bar{\alpha}}{\sqrt{b^{2}-s^{2}}}-\left(b \sigma_{1}\right) u_{A}, \quad s_{1}=0, \quad s_{A}=-\frac{1}{2} \sigma_{A} b^{2}, \\
& s_{10}=\frac{1}{2} b \overline{\sigma_{0}}, \quad s_{A 0}=\frac{1}{2} \frac{\sigma_{A} b s \bar{\alpha}}{\sqrt{b^{2}-s^{2}}} \\
& h_{1}=b-\frac{s^{2}}{b}, \quad h_{A}=-\frac{\sqrt{b^{2}-s^{2}} s u_{A}}{b \bar{\alpha}} .
\end{aligned}
$$

Proof of Theorem 1.1: We remark that $\tilde{b}=$ constant. If $\tilde{b}=0$, then $F=e^{k(x)} \tilde{\alpha}$ is a Riemannian metric. Now, let $F$ is not Riemannian metric. Assume that $F$ is a conformally flat $(\alpha, \beta)$-metric with relatively isotropic 
mean Landsberg curvature. By (3.2) and $r_{0}+s_{0}=0$, we get

$\frac{\alpha^{2}}{b^{2}-s^{2}}\left[\Psi_{1}+s \frac{\Phi}{\Delta}\right]\left(r_{00}-2 \alpha Q s_{0}\right) h_{j}+\alpha\left[-\alpha^{2} Q^{\prime} s_{0} h_{j}+\alpha Q\left(\alpha^{2} s_{j}-y_{j} s_{0}\right)\right.$

$\left.+\alpha^{2} \Delta s_{j 0}+\alpha^{2}\left(r_{j 0}-2 \alpha Q s_{j}\right)-\left(r_{00}-2 \alpha Q s_{0}\right) y_{j}\right] \frac{\Phi}{\Delta}+c(x) \alpha^{4} \Phi\left(\phi-s \phi^{\prime}\right) h_{j}=0$.

Letting $j=1$ in (3.15), we have

$\frac{\alpha^{2}}{b^{2}-s_{2}}\left[\Psi_{1}+s \frac{\Phi}{\Delta}\right]\left(r_{00}-2 \alpha Q s_{0}\right) h_{1}+\alpha\left[-\alpha^{2} Q^{\prime} s_{0} h_{1}+\alpha Q\left(\alpha^{2} s_{1}-y_{1} s_{0}\right)\right.$

$\left.+\alpha^{2} \Delta s_{10}+\alpha^{2}\left(r_{10}-2 \alpha Q s_{1}\right)-\left(r_{00}-2 \alpha Q s_{0}\right) y_{1}\right] \frac{\Phi}{\Delta}+c(x) \alpha^{4} \Phi\left(\phi-s \phi^{\prime}\right) h_{1}=0$.

Putting (3.10)-(3.14) into (3.16) and multiplying the result with $2 \Delta\left(b^{2}-\right.$ $\left.s^{2}\right)^{5 / 2}$ implies that

$2 b^{2}\left(b^{2}-s^{2}\right)^{3 / 2} \Delta\left(b \Phi \phi c-b \Phi s \phi^{\prime} c-\Psi_{1} \sigma_{1}\right) \bar{\alpha}^{4}+b^{2}\left(b^{2}-s^{2}\right) \bar{\sigma}_{0}\left(b^{4} \Phi Q^{\prime}-b^{2} \Phi \Delta-b^{2} \Phi Q^{\prime} s^{2}\right)$

$\left.+2 b^{2} \Psi_{1} \Delta Q+b^{2} \Phi+b^{2} \Phi Q s+2 \Psi_{1} \Delta s\right) \bar{\alpha}^{3}=0$.

From (3.17), we get

$\Delta\left[b \Phi \phi c-b \Phi s \phi^{\prime} c-\Psi_{1} \sigma_{1}\right]=0$,

$$
\left.\bar{\sigma}_{0}\left(b^{4} \Phi Q^{\prime}-b^{2} \Phi \Delta-b^{2} \Phi Q^{\prime} s^{2}\right)+2 b^{2} \Psi_{1} \Delta Q+b^{2} \Phi+b^{2} \Phi Q s+2 \Psi_{1} \Delta s\right)=0 .
$$

Note that $\Delta=Q^{\prime}\left(b^{2}-s^{2}\right)+s Q+1$. Simplifying (3.19) yields

$$
\left(b^{2} \Psi_{1} \Delta Q+\Psi_{1} \Delta s\right) \overline{\sigma_{0}}=0,
$$

that is

$$
\Psi_{1} \Delta\left(b^{2} Q+s\right) \overline{\sigma_{0}}=0 .
$$

Letting $j=A$ in (3.15), we have

$$
\frac{\alpha^{2}}{b^{2}-s^{2}}\left[\Psi_{1}+s \frac{\Phi}{\Delta}\right]\left(r_{00}-2 \alpha Q s_{0}\right) h_{A}+\alpha\left[-\alpha^{2} Q^{\prime} s_{0} h_{A}+\alpha Q\left(\alpha^{2} s_{A}-y_{A} s_{0}\right)\right.
$$

$$
\left.+\alpha^{2} \Delta s_{A 0}+\alpha^{2}\left(r_{A 0}-2 \alpha Q s_{A}\right)-\left(r_{00}-2 \alpha Q s_{0}\right) y_{A}\right] \frac{\Phi}{\Delta}+c(x) \alpha^{4} \Phi\left(\phi-s \phi^{\prime}\right) h_{A}=0 .
$$

Putting (3.10)-(3.14) into (3.21) and by using the similar method used in the case of $j=1$, we get

$-\left(s \Delta+s+b^{2} Q\right) b^{2} \Phi \sigma_{A} \bar{\alpha}^{2}+\left[\left(s \Delta+s+b^{2} Q\right) b^{2} \Phi+2 s\left(b^{2} Q+s\right) \Psi_{1} \Delta\right] \bar{\sigma}_{0} u_{A}=0$,

$$
s\left(b^{2}-s^{2}\right)\left[b\left(\phi-s \phi^{\prime}\right) \Phi c-\Psi_{1} \sigma_{1}\right] \Delta u_{A}=0 .
$$


It is easy to see that (3.23) is equivalent to (3.18). Further, multiplying (3.22) with $u^{A}$ implies that

$$
s\left(b^{2} Q+s\right) \Psi_{1} \Delta \bar{\sigma}_{0} \bar{\alpha}^{2}=0 .
$$

It is easy to see that (3.24) is equivalent to (3.20). In summary, conformally flat $(\alpha, \beta)$-metrics with relatively isotropic mean Landsberg curvature satisfy (3.18) and (3.20). According to (3.20), we have some cases as follows:

Case (i): If $b^{2} Q+s=0$, then we have $\phi=\kappa \sqrt{b^{2}-s^{2}}$, which is a contradiction with the assumption that $\phi=\phi(s)$ is $\phi(s)=\sqrt[4]{a_{1}+a_{2} s^{2}+a_{3} s^{4}}$, Then we have $b^{2} Q+s \neq 0$.

Case (ii): If $\Psi_{1}=0$, then by Lemma 3.4, $F$ is Riemannian.

Case (iii): If $\Psi_{1} \neq 0$, then $\sigma_{A}=0$. In the following, we prove that if $\Psi_{1} \neq 0$, then by (3.18) one can get $\sigma_{1}=0$.

Assume that

$$
\phi=\sqrt[4]{a_{1}+a_{2} s^{2}+a_{3} s^{4}}, \quad 4 a_{1} a_{3}-a_{2}^{2} \neq 0
$$

here $a_{1}, a_{2}, a_{3}$ are numbers independent of $s$ and $a_{i} \neq 0, i=1,2,3$. Simplifying (3.18) and multiplying it by $\Delta^{2}$, we get

$$
\left\{\left[-s \Phi+\left(b^{2}-s^{2}\right) \Phi^{\prime}\right] \Delta-\frac{3}{2}\left(b^{2}-s^{2}\right) \Phi \Delta^{\prime}\right\} \sigma_{1}-b \Delta^{2} \Phi\left(\phi-s \phi^{\prime}\right) c=0:
$$

Putting (3.25) into (3.26) and multiplying it by

$$
-\left(a_{2} s^{2}+2 a_{1}\right) \sqrt[14]{\left(a_{1}+a_{2} s^{2}+a_{3} s^{4}\right)^{3}}
$$


by using maple program, we can obtain the following

$$
\begin{aligned}
& 2\left[\left((n-8) b^{2}-3 n s^{2}+6 s^{2}\right) b^{2} a_{2}^{5} s^{10}+\left\{(-n+8) b^{6} a_{1} s^{6}+(9 n-72) b^{4} a_{1} s^{8}+(-16 n+128) a_{1} b^{2} s^{10}\right.\right. \\
& \left.+(24-48 n) s^{12} a_{1}-\left((n-8) b^{2}-5 n s^{2}+4 s^{2}\right) b^{4} a_{3} s^{10}\right\} a_{2}^{4}+2\left[\left\{(4 n+7) b^{6} s^{4}-(10 n+160) b^{4} s^{6}\right.\right. \\
& \left.-(9 n-210) b^{2} s^{8}+(40 n-32) s^{10}\right\} a_{1}^{2}+\left(3(n-8) b^{4}+(-2 n+16) b^{2} s^{2}+(13 n+22) s^{4}\right) b^{2} a_{1} a_{3} s^{8} \\
& \left.+(n+1) b^{6} s^{12} a_{3}^{2}\right] a_{2}^{3}+4 s^{2}\left\{\left(-(3 n+18) b^{6}-(3 n+141) b^{4} s^{2}+(94-11) b^{2} s^{4}+(36 n+48) s^{6}\right) a_{1}^{2}\right. \\
& \left.s^{4}\left((5 n-82) b^{6}+(83 n-94) b^{2} s^{4}-(28 n-80) s^{6}\right) a_{3} a_{1}+\left(2(n-8) b^{2}+(n+19) s^{2}\right) b^{4} a_{3}^{2} s^{8}\right\} a_{1} a_{2}^{2} \\
& -8\left[\left((6 n+18) b^{4} s^{2}+(3 n+42) b^{2} s^{4}-(16 n-64) s^{6}-3 b^{6}\right) a_{1}^{2}+\left[(15 n+36) b^{6}+(2 n-52) b^{4} s^{2}\right.\right. \\
& \left.\left.+3(34-17) b^{2} s^{4}+3(8 n-32) s^{6}\right] a_{3} a_{1} s^{4}+\left[(9 n+15) b^{4}+(2-2 n) b^{2} s^{2}+10(n-2) s^{4}\right] b^{2} a_{3}^{2} s^{8}\right] a_{1}^{2} a_{2} \\
& \left.+\left(6 b^{2} a_{1} a_{3}-\frac{1}{2} b^{2} a_{2}^{2}+2 a_{1} a_{2}\right) s^{2}+a_{1}\left(a_{2} b^{2}+2 a_{1}\right)\right) \cdot \\
& \left.+\left(4(n-5) b^{2} a_{2}+8(n+4) a_{1}\right) a_{1} s^{2}-24 b^{2} a_{1}^{2}\right\}\left(4 a_{1} a_{3}-a_{2}^{2}\right)\left(a_{2} s^{2}+2 a_{1}\right)^{7}\left(\left(a_{2} a_{3} b^{2}-4 a_{1} a_{3}+\frac{3}{2} a_{2}^{2}\right) s^{4}\right. \\
& +\left[\left((3 n+18) b^{2} s^{2}-(4 n-16) s^{4}\right) a_{1}^{2}+\left[(15 n-54) b^{4} s^{2}+(82-29 n) b^{2} s^{4}+(12 n-48) s^{6}\right] a_{1} a_{3} s^{2}\right. \\
& \left.\left.+\left[18(n-1) b^{6}+(69-45 n) b^{4} s^{2}+(34 n-68) b^{2} s^{4}-(8 n+16) s^{6}\right] a_{3}^{2} s^{6}\right\} a_{1}^{3}\right]\left(4 a_{1} a_{3}-a_{2}^{2}\right) \sigma_{1} \\
& +\left\{\left(6 n a_{2}^{2}+4 a_{2} a_{3} b^{2}(n+1)-16 a_{1} a_{3}(n-2)\right) s^{6}+\left((4-2 n) a_{2}^{2} b^{2}+8(n+4) a_{1} a_{2}+24 n b^{2} a_{1} a_{3}\right) s^{4}\right. \\
& +28)
\end{aligned}
$$

Let us put

$$
\nu:=\frac{1}{\left(4 a_{1} a_{3}-a_{2}^{2}\right)} .
$$

Multiplying (3.28) with $\nu$ implies that

$$
\Pi_{1} s^{25}+\Pi_{2} s^{24}+\ldots+\Pi_{25} s+\Pi_{26}=0,
$$


where $\Pi_{i}(1 \leq i \leq 25)$, are polynomials of $a_{1}, a_{2}, a_{3}, b, c$, and $\sigma_{1}$. Equation (3.29) is equivalent to the following two equations

$$
\begin{aligned}
& \Pi_{1} s^{25}+\Pi_{3} s^{23}+\ldots+\Pi_{23} s^{3}+\Pi_{25} s=0, \\
& \Pi_{2} s^{24}+\Pi_{4} s^{22}+\ldots+\Pi_{24} s^{2}+\Pi_{26}=0,
\end{aligned}
$$

where $\Pi_{26}=96 b^{4} a_{1}^{5} \sigma_{1}$. (3.31) implies that $\Pi_{26}=0$, because $b \neq 0$ and $a_{1} \neq 0$, then $\Pi_{26}=0$ implies that $\sigma_{1}=0$. Together with $A=0$, it follows that $\sigma$ is a constant, which means that $F$ is a locally Minkowski metric. This completes the proof.

\section{REFERENCES}

[1] P.L. Antonelli, R.S. Ingarden and M. Matsumoto, The Theory of Sprays and Finsler Spaces with Applications in Physics and Biology, Kluwer Academic Publishers, Dordrecht, 1993.

[2] G.S. Asanov, Finslerian metric functions over product $R \times M$ and their potential applications, Rep. Math. Phys. 41(1998), 117-132.

[3] X. Cheng, H. Wang and M. Wang, $(\alpha, \beta)$-metrics with relatively isotropic mean Landsberg curvature, Publ. Math. Debrecen, 72(2008), 475-485.

[4] S. S. Chern and Z. Shen, Riemann-Finsler Geometry, World Scientiflc, Singapore, 2005.

[5] B. Li and Z. Shen, On a class of weak Landsberg metrics, Science in China Series A, $\mathbf{5 0}(2007), 75-85$.

[6] A. Tayebi, On generalized 4-th root metrics of isotropic scalar curvature, Mathematica Slovaca, 68(2018), 907-928.

[7] A. Tayebi and B. Najafi, On m-th root Finsler metrics, J. Geom. Phys. 61(2011), 1479-1484.

[8] A. Tayebi and B. Najafi, On m-th root metrics with special curvature properties, C. R. Acad. Sci. Paris, Ser. I. 349(2011), 691-693.

[9] A. Tayebi and M. Razgordani, On conformally flat fourth root $(\alpha, \beta)$-metrics, Differ. Geom. Appl. 62(2019), 253-266.

[10] A. Tayebi and M. Shahbazi Nia, A new class of projectively flat Finsler metrics with constant flag curvature $\mathbf{K}=1$, Differ. Geom. Appl. 41(2015), 123-133.

(Akbar Tayebi) Department of Mathematics, Faculty of Science, University OF QOM, QOM, IRAN.

Email address: akbar.tayebi@gmail.com

(Marzeiya Amini) Department of Mathematics, Faculty of Science, University OF QOM, QOM, IRAN.

Email address: marzeia.amini@gmail.com 